\title{
ANÁLISIS E INTEGRACIÓN DE LA CADENA DE SUMINISTRO PARA EVITAR EL EFECTO LÁTIGO
}

\section{ANALYSIS AND INTEGRATION OF THE SUPPLY CHAIN TO AVOID THE WHIP EFFECT}

\author{
Nazarena Noemí Lazala Diaz ${ }^{1}$ \\ Víctor Gisbert Soler ${ }^{2}$ \\ Ana Isabel Pérez Molina ${ }^{3}$
}

1. Graduada en Ingeniería Industrial en República Dominicana. Máster en Ingeniería en Organización y Logística. Universidad Politécnica de Valencia, España. E-mail: naladia@epsa.upv.es

2. Doctor Ingeniero Industrial. Departamento de Estadística e Investigación Operativa Aplicadas y Calidad. Universidad Politécnica de Valencia. E-mail: v.gisber@eio.upv.es

3. Ingeniera Técnico Industrial Química. Ingeniera en Organización Industrial. Doctor por la Universidad Politécnica de Valencia. Departamento de Estadística e Investigación Operativa Aplicadas y Calidad. Universidad Politécnica de Valencia, España. E-mail: anpemo@eio.upv.es

\section{Citación sugerida:}

Lazala Diaz, N., Gisbert Soler, V. y Pérez Molina, A.I. (2017). Análisis e integración de la cadena de suministro para evitar el efecto látigo. 3C Empresa: investigación y pensamiento crítico, Edición Especial, 19-28. DOI: <http://dx.doi.org/10.17993/3cemp.2017.especial.19-28/>. 


\section{RESUMEN}

La cadena de suministro, conocida por sus siglas en ingles SCM (Supply Chain Managment), está conformada por distintos niveles de acuerdo a su estructura y tamaño. En este artículo se presentan y analizan las incidencias que tiene el efecto látigo (que no es más que la amplificación de la demanda, donde mientras más alto es el nivel, mayor la amplificación) sobre la misma, conociendo sus causas y consecuencias, posteriormente se presentan soluciones que garanticen la integración de la cadena de suministro, permitiendo que los flujos, tanto de información como de material, se realicen de manera óptima.

\section{ABSTRACT}

The supply chain, known by its abbreviations in English SCM (Supply Chain Management), is conformed by different levels according to their structure and size. This article presents and analyzes the effects of the whip effect (which is no more than the amplification of demand, where the higher the level, the greater the amplification) on the same, knowing its causes and consequences, Present solutions that guarantee the integration of the supply chain, allowing the flows, both information and material, to be carried out in an optimal way.

\section{PALABRAS CLAVE}

Suministro, Inventario, Efecto látigo, Integración, Cliente, Proveedor.

\section{KEY WORDS}

Supply, Inventory, Bullwhip, Integration, Client, Provider. 


\section{INTRODUCCIÓN}

La gestión moderna de las cadenas de suministro considera la premisa de que los miembros de la cadena están primordialmente interesados en optimizar sus propios objetivos. Es principalmente por el autoabastecimiento y la búsqueda del bien singular, que surgen efectos como el Bullwhip, ya que, buscando seguridad, en cada nivel de la cadena se produce una amplificación de pedidos (aguas arriba) generando grandes ineficiencias en toda la cadena de suministro. Los síntomas de dicha distorsión se traducen en niveles de inventario excesivos, capacidad insuficiente o excesiva, escaso servicio al cliente, previsiones erróneas de la demanda del mercado, planos de producción inciertos, etc. Se ha estimado que las consecuencias económicas del efecto bullwhip pueden suponer hasta un $30 \%$ de aumento en costes innecesarios para una empresa (Metters 1997).

Este articulo presenta un estudio de las transformaciones que sufren los pedidos a lo largo de la cadena de suministro, analizando cada una de las causas o factores que inciden en que se produzca ese comportamiento en los distintos niveles, para posteriormente proponer algunas alternativas de mejora que, con su implementación, nos permita disminuir considerablemente las ineficiencias provocadas por el efecto látigo en la cadena de suministro, adoptando para ello un sistema de métricas para evaluar los beneficios internos de la cadena, medidos en términos de la estabilidad de la orden de pedido y de los inventarios, de la robustez del sistema y del nivel de cumplimento de la demanda del mercado.

El presente artículo está organizado de la siguiente manera:

- Antecedentes principales del tema en cuestión

- Desarrollo y metodología

- Resultados

- Conclusiones

Además, una sección de fuentes bibliográficas que le permita al lector indagar más sobre el tema.

\section{ANTECEDENTES}

Varios autores han descrito y clasificado sus enfoques al problema de la amplificación de la demanda y de la inestabilidad de la cadena de suministro, entre los principales podemos destacar:

El industrial Jay Forrester, creador de la dinámica de sistemas, demostró la posibilidad de que la demanda cursada de un comprador a su proveedor incrementa su amplitud a medida que se remonta aguas arriba de la Cadena de suministro en 1951. El enfoque de este antecedente se caracteriza por que explica cómo la demanda del cliente va 
trasladándose de forma cada vez más distorsionada a través de la cadena de suministro hasta el fabricante.

Herlyn, W., "El Bullwhip Efecto en Cadenas de Suministro expandido y el Concepto de Cantidades Acumulables", Este antecedente se relaciona al enfoque expresado por el industrial Jay Forrester en que explican que a lo largo de la cadena de suministro se pueden generar en algunos niveles rotura de stock y en otro un exceso de inventario debido a las cantidades acumuladas con el objetivo de buscar seguridad. Este se caracteriza por que explica como el efecto látigo a medida que se expande en la cadena de suministro, genera cada vez más cantidades acumuladas debido a la poca integración entre los niveles.

Dentro de los estudios realizados, se destacan varias líneas de actuación, de las cuales podemos mencionar:

Las originadas por la fusión de las teorías enunciadas en los trabajos de Forrester y del premio Nobel de Economía Simón (1978), este antecedente se caracteriza por que se apoyan en las técnicas propias de la ingeniería de control para analizar el comportamiento de la gestión del inventario y Las basadas en la simulación de sistemas, entre las que destaca el "Juego de la Cerveza", método ideado por Sterman, que se caracteriza porque muestra de una forma sencilla la creación del efecto látigo como una consecuencia de una inapropiada sincronización de la información de corto plazo (Aproximadamente un horizonte de planificación de doce semanas) en la cadena de suministro por parte de sus componentes.

Todos los antecedentes mencionados anteriormente se relacionan por que buscan explicar, con diferentes enfoques, que la cadena de suministro se ve afectada por el Bullwhip, cuando entre los diferentes niveles, no se cuenta con la integración necesaria para evitar la incertidumbre, que, a raíz de esto, los mismos distorsionan la demanda real con el objetivo de tener un colchón de seguridad.

\section{3. ¿QUÉ ES BULLWHIP?}

El efecto látigo se define como la amplificación de la demanda aguas arriba a lo largo de la cadena de suministro cuando existe una variación en la demanda del mercado, o incluso aun, cuando la demanda es estable, lo que provoca una frecuente inestabilidad de los inventarios, y esto genera a su vez, costes innecesarios en cada una de las empresas afectadas. Es decir, cuando tenemos una cadena de suministro conformada por una empresa o minorista, un proveedor nivel 1, un proveedor nivel 2 (proveedor del Proveedor 1) y un proveedor nivel 3 (proveedor del proveedor nivel 2), la empresa o minorista toma decisiones ajustando sus pedidos en base a una demanda real, y ésta con el fin de satisfacer $100 \%$ la demanda del cliente, hace un pedido a su proveedor aumentando en una proporción racional (de acuerdo a los factores, naturaleza o estrategia empresarial) la cantidad realmente demandada, creando un colchón de seguridad para responder a situaciones inestables, el problema radica en que cada vez que el pedido llega a un nivel de la cadena, este va propagándose en aumento, tal como podemos ver gráficamente en la 
Figura 1.

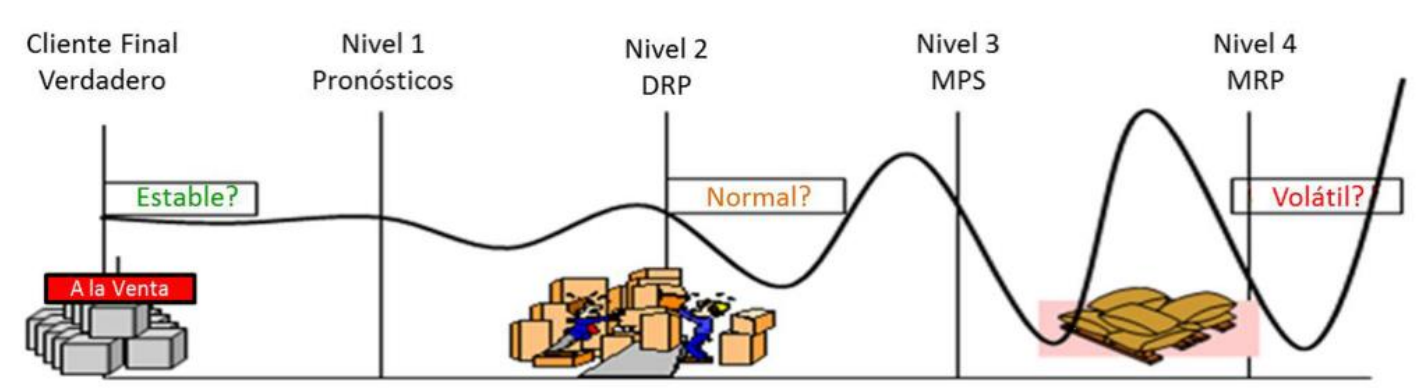

Figura 1. Efecto Látigo.

Las principales causas de que, en cada uno de los niveles de la cadena, la demanda sufra unas actualizaciones en sentido mayor, vienen dadas principalmente por:

\section{- La incertidumbre}

Esta situación se presenta cuando no se dispone de la misma información en los distintos niveles de la cadena, y, por ende, se trabaja bajo incertidumbre.

\section{- Poca comunicación o desconfianza}

En el ámbito de negocios, muchas veces desconfiamos de nuestros proveedores por la misma lucha de poderes entre compradores y vendedores, quienes entienden que cada una de las partes está en busca del beneficio individual.

\section{- Aprovechamiento de ofertas o variación de los precios}

Las empresas, buscando mejores precios para ser más competitivos en el mercado, buscan costes más bajos y en este intento, cuando se ven frente a una oferta tratan de aprovecharla al máximo, pidiendo grandes lotes de mercancía con el objetivo de que este "precio de oferta" sea duradero para sus actividades productivas. Además, cuando se ven frente a un mercado con grandes fluctuaciones en los precios, compran grandes lotes, así cuando los enfrente el próximo aumento de precio, aun tendrán mercancía con el precio anterior.

\section{- Rapidez en el procesamiento de ordenes}

Nos encontramos en un mundo empresarial muy competitivo, donde se busca tener prioridad en el ámbito de actividad productiva que se desempeña. Es por ello, que se busca que los proveedores respondan con la mayor rapidez, evitando verse en la situación de tener que retrasar pedidos o incumplir a un cliente, las empresas realizan pedidos en el momento que no lo necesitan para que cuando realmente se necesite, ya esté disponible.

Este fenómeno genera diversas consecuencias en las empresas que lo padecen y en la cadena de suministro de manera global. Dentro de las principales podemos destacar las siguientes: 
- Ineficiencia en los procesos

- Aumento en los costes de almacenamiento y producción

- Acumulación de inventario

\section{METODOLOGÍA}

Luego de todo lo analizado en las fases anteriores, se presentan varias propuestas a implementar en las empresas, con el fin de neutralizar esa deficiencia en la cadena de suministro, eliminarla y evitarla. Estos puntos de acción van directamente relacionado a las causas del Bullwhip, ya que al combatir las mismas, estaremos reduciendo el efecto látigo en la cadena hasta lograr eliminarla. De acuerdo a algunos de los antecedentes estudiados, (Riddalls et al. 2000, Disney et al. 2004, Kleijnen y Smits 2003, Dejonckheere et al. 2004, Holweg y Disney 2005, Geary et al. 2006, Towill et al. 2007), En 1993 van Ackere et al. (1993) proporcionaron un marco útil para clasificar las medidas que se pueden utilizar en una cadena de suministro con el objeto de reducir o evitar el efecto bullwhip. Esta metodología de acción se menciona a continuación;

Compartir información en toda la cadena porque a pesar de que existe demanda en todos los niveles, la demanda que realmente importa es la del mercado o cliente final. Cada escalón debe conocer la demanda del cliente consumidor del producto final y no solo de los pedidos realizados por su cliente directo (la empresa). Para ello, es importante que cada uno de los niveles entienda que necesitan de la integración de toda la cadena para lograr resultados esperados.

Una estrategia que podemos aplicar y obtener buenos resultados es reducir la cantidad de proveedores, que por consiguiente también reduciría la cantidad de niveles de la cadena. Al tener proveedores limitados, justo los necesarios, esto simplificaría la complicación, disminuyendo la oscilación que crea el efecto látigo y mejoraría en gran medida la comunicación. Una opción sería crear un centro de distribución que es quien se encarga de recibir y enviar todos pedidos a la empresa o minorista, agrupando todos los pedidos de los diferentes proveedores en un solo, para ser enviado, de modo que el minorista se comunique directamente con el centro de distribución y este a su vez, con todos los demás proveedores que se encuentran aguas arriba.

Establecer contratos de compra a largo plazo. Las empresas Lean ven a sus proveedores como parte de su empresa en cierto modo y esta es una forma de hacer sentir a los proveedores partes de nuestro sistema estableciendo basado en objetivos compartidos $y$ normas de funcionamiento común. Además, esta metodología generaría un alto nivel de confianza proveedor-comprador, puesto que se verá más comprometido y con más interés de realizar inversiones de mejoras a largo plazo ya que le garantiza tener un flujo de ingresos disponible en ambas partes.

Mejorar los procesos operativos. Reduciendo los tiempos de entrega tendremos un impacto directo en los inventarios, puesto que mientras más largo es el tiempo de entrega, 
más difícil es coordinar un stock de seguridad y la producción, es por ello que cuando nos vemos frente a esta situación (tiempo de suministro muy largo), las empresas tienen a pedir lotes en grandes cantidades que en realidad no necesitan, en fin de asegurar inventario para la realización de sus actividades productivas, pero esta situación genera ineficiencia en el proceso.

Reducir la variabilidad de la demanda. Una buena estrategia para mantener una demanda constante o poco variable, es establecer una política de precios bajos al consumidor, con lo cual, los clientes se sentirán identificados con la empresa y confiará en sus precios, por lo que la demanda se mantendrá establece o parecida al histórico.

Aumentar el nivel de comunicación y el flujo de información. Es importante que las decisiones que se tomen en cada eslabón de la cadena correspondan a un mismo criterio. Evitar la unilateralidad en la toma de decisiones es posible si se tiene una visión global de la cadena de suministro y aplicar un cuadro de mando es imprescindible como herramienta para alcanzar este objetivo.

\section{RESULTADOS}

Al implementar cada uno de las propuestas desarrolladas en la metodología, obtendremos unos resultados bastante notorios que lograran neutralizar los efectos del Bullwhip y en el mejor de los casos, eliminarlo por completo. A continuación, se presenta una pequeña tabla en modo resumen, que muestra los resultados más relevantes que se obtienen al implementar la metodología propuesta en la cadena de suministro.

\begin{tabular}{|c|c|c|}
\hline \multicolumn{3}{|c|}{ Resultados al implementar metodología propuesta } \\
\hline En relación a & Antes & Después \\
\hline Inventario & $\begin{array}{c}\text { Tendencia a la compra de grandes } \\
\text { lotes y al almacenamiento }\end{array}$ & $\begin{array}{c}\text { Compra en pequeños lotes con } \\
\text { mayor frecuencia y JIT }\end{array}$ \\
\hline & $\begin{array}{l}\text { - } \quad \text { Muchos proveedores } \\
\text { - } \quad \text { Selección basada en los } \\
\text { precios }\end{array}$ & $\begin{array}{l}\text { - Pocos proveedores } \\
\text { - } \quad \text { Evaluación y homologación }\end{array}$ \\
\hline Proveedores & - No se comparte información & $\begin{array}{l}\text { - Se comparte información } \\
\text { de manera abierta } \\
\text { - El servicio como método de } \\
\text { selección }\end{array}$ \\
\hline Optimización & Optimización local o individual & Optimización global \\
\hline Planificación & Poca y a corto plazo & ERP integrado y Largo plazo \\
\hline
\end{tabular}




\begin{tabular}{|c|c|c|}
\hline Calidad & $\begin{array}{l}\text { Control de calidad de los artículos } \\
\text { comprados }\end{array}$ & $\begin{array}{l}\text { - Acuerdos de calidad } \\
\text { concertada } \\
\text { - Co-fabricación y gestión } \\
\text { coordinada de inventarios }\end{array}$ \\
\hline Comunicación & Poca y lenta & Rápida y fluida \\
\hline Transporte & En manos del proveedor & Coordinada y planificada \\
\hline Diseño & No se cuenta con el proveedor & $\begin{array}{l}\text { Diseño colaborativo e integración de } \\
\text { tecnologías y procesos }\end{array}$ \\
\hline Relaciones & Gran desconfianza & $\begin{array}{c}\text { A largo plazo y orientadas a la } \\
\text { integración, basadas en el bien } \\
\text { común }\end{array}$ \\
\hline
\end{tabular}

Tabla 1. Resultados obtenidos.

\section{CONCLUSIONES}

Finalmente, a modo de conclusión, se expresaron en las líneas anteriores todas las observaciones, causas y consecuencias del Bullwhip, fenómeno presentado en las cadenas de suministros cuando no se cuenta con la integración suficiente de la misma. Luego de analizar todo ello y comprender el modo en el que funciona el mismo, también se presentaron una serie de herramientas y/o metodologías que las empresas pueden implementar para neutralizar o evitar la gran ineficiencia que este produce a la cadena de suministro de manera global.

El efecto látigo se refiere a la transformación sufrida por la demanda del mercado a medida que se transmite desde los niveles más cercanos al cliente final hacia los más alejados en la cadena de suministros. Las decisiones, basadas en la información recibida, no se corresponden con la realidad del mercado y las ineficiencias resultantes van en aumento.

El objetivo del presente artículo ha sido estudiar el impacto del efecto látigo en las cadenas de suministro desde cada uno de sus niveles, para posteriormente, analizar cuáles son las diferentes alternativas de mejora que podemos aplicar con el fin de disminuir la ocurrencia del mismo, o en el mejor de los casos, eliminarlo.

Es importante destacar, que las ineficiencias de la cadena, viene determinada por el modo de acción y las decisiones tomadas por cada uno de los agentes que la conforman, es por ello, que se ha resaltado durante el análisis, lo vital que resulta para el éxito operativo de la cadena de suministro, la integración de esta. Para ello, enumeramos algunas de las conclusiones obtenidas durante el artículo: 
La base del éxito en una cadena de suministro es dejar de pensar de manera local o independiente. Hay que reconocer a cada uno de los agentes de la misma como una extensión de nuestra empresa.

Una vez implementadas las metodologías propuestas, la cadena de suministro aumentara su eficiencia operativa, ya que, al contar con un flujo de información adecuado y actualizado, permitirá que se tomen las decisiones certeras, basadas en criterios previamente establecidos, fomentando el bien común en toda la cadena. 


\section{REFERENCIAS BIBLIOGRÁFICAS}

Bray, Robert L., y Haim Mendelson. "Transmisión de información y el bullwhip efecto: Una investigación empírica." Ciencia de administración 58.5 (2012): 860-875.

Disney, S.M., y Towill, D.R. (2003). En el bullwhip y varianza de inventario producida por una política de ordenar. Omega, la Revista Internacional de Ciencia de Administración, 31 (3), 157-167.

Herlyn, W., "El Bullwhip Efecto en Cadenas de Suministro expandido y el Concepto de Cantidades Acumulables", en: Blecker et al. (Eds.): "Métodos innovadores en Logísticas y Cadena de Suministro Administración", p. 513-528, epubli GmbH, Berlín, 2014, ISBN 978-3-8442-9878-9

Juan Carlos Mejía Villamizar, (22 de enero 2014), Efecto látigo en la planeación de la cadena de abastecimiento, medición y control. Obtenido de http://www.scielo.org.co/pdf/cein/v23n2/v23n2a03.pdf

Lee, H.L., Padmanabhan, V., Whang, S.: Information distortion in a supply chain: the bullwhip effect. Management Science 43, pp. 546-58 (1997a).

Roman Mestre (24 octubre 2011), Historias del PDD XXI, El efecto Bullwhip. Obtenido de https://romanmestre.com/2011/10/24/historias-del-pdd-xxi-el-efecto-bullwhip/

Ros Mcdonnell, L., et al, (2006). Variabilidad de la demanda y sus efectos según distintas políticas de gestión de la Cadena de Suministro. Modelado y Simulación. En: http://www.adingor.es/Documentación/CIO/cio2006/docs/000082_final. pdf (febrero del 2012). 